\title{
A SLIT RETINOSCOPE Slit disc for use in connexion with mirror for retinoscopy
}

B Y

\section{KLEIN. \\ LONDON}

The Priestley Smith mirror has been used extensively for retinoscopy. The model described here is similar to the original and differs only in that both plane and concave mirrors are two sided, one side of each being covered with a disc in which there is a narrow oblong aperture. The disc can be revolved so that the aperture can be made to run in any direction. The disc covered side thus becomes a narrow oblong mirror, reflecting a strip of light the direction of which can be turned in any meridian by revolving the slit-disc. The instrument can be used advantageously in all cases of retinoscopy, especially in cases of astigmatism.*

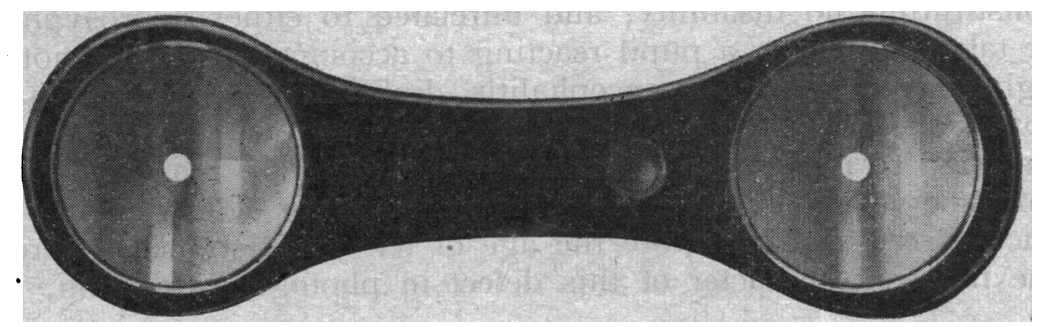

FIG. 1 .

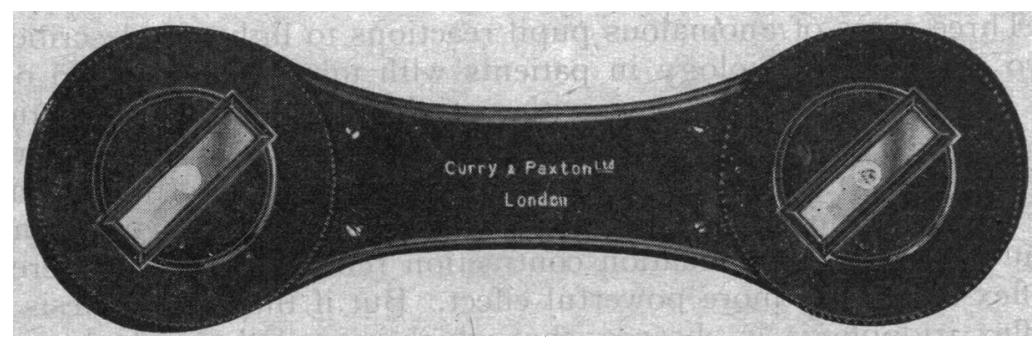

FIG. 2.

Method of examination: Whether plane or concave mirror is used is a matter of choice. The position of the lamp: This has no influence when the plane mirror is used, but with the concave mirror, the lamp should be on the side, or above, and a little

* The mirrors have a central non-perforating hole of $1.3 \mathrm{~mm}$. diameter. 
behind the patient's head, about $125-150$ centimetres from the observer's mirror. The smallest opening of the Lister ophthalmoscopic lamp is quite satisfactory but any small circular light source is adequate.

The examination is begun with the mirror side, correcting lenses being placed before the patient's eye in the usual way, until the neutral point is nearly reached. Then the slit side is turned towards the patient and the final adjustment of the correction is made. In case of astigmatism the slit should be brought in the meridian first refracted. For example, in a compound hypermetropic astigmatism " with the rule," the position of the slit should be vertical. When the refraction in this meridian has been determined, the slit is put at right angles to the meridian examined, in our example at $180^{\circ}$, and in case of astigmatism it will be found that the shadow has a sharply defined edge. If the slit is not in the direction of the corresponding meridian of the astigmatism, the edge of the shadow becomes ill defined. This enables us to find the meridian of the astigmatism by rotating the slit into a position where the shadow edge becomes sharpest. Now comes the correction of the astigmatism. For this purpose we leave the slit in the meridian determined, and place further lenses before the eye until the neutral point is reached. This may be done with spherical or cylindrical glasses. Though the latter may be regarded as the more satisfactory, they have to be placed in the right meridian, and this prolongs the examination, which in the pressure of work of an outpatient department is a great disadvantage, and spherical glasses meet the need in most cases.

At first sight it might appear that the slit retinoscope is like a streak retinoscope, except that one is a reflecting the other a luminous instrument. The relation between these two types of instruments can be better appreciated if we consider the optics of retinoscopy the elements of which are: light source, apertures (such as the pupil, the mirror), optical surfaces and the screen (retina). Of these the first two can be changed. In the case of streak retinoscopy the light source is altered to an oblong one. In the slit retinoscope it is one of the apertures-that is the mirror itself-is altered to an oblong one, and the form of the light source is unchanged. It is common to both methods that the oblong element is present and can be rotated into different axes.

The instrument is manufactured by Messrs. Curry \& Paxton Ltd., I.ondon, W.1.

\section{REFERENCE}

J. N. Evans (1940).-Amer. Jl. Ophthal., Series 3, Vol. XXIII, p. 1158. 